Ann. Sci. forest., 1980, 37 (1), 85-89.

\title{
Etude de la survie des uredospores de Melampsora larici-populina kleb et de M. Allii-populina Kleb
}

\author{
J. PINON \\ Laboratoire de Pathologie forestière \\ Centre national de recherches forestières, I.N.R.A., \\ Champenoux, 54280 Seichamps
}

\section{Résumé}

Le pouvoir germinatif des urédospores de $M$. Larici-populina et de $M$. Allii-populina conservées au laboratoire à $+4^{\circ} \mathrm{C}$ décroît régulièrement et, comme en pépinière, tend à s'annuler après 7 ou 8 mois. Si l'humidité relative est faible $(34$ p. 100$)$ la conservation est portée à un an ef les sporées germent à plus de 50 p. 100 durant les huit premiers mois.

\section{Introduction}

Taris (1968) a montré qu'en nature un faible pourcentage d'urédospores de M. Larici-populina était encore apte à germer au printemps qui suit leur différenciation. Nous avons voulu étudier au laboratoire l'évolution dans le temps du pouvoir germinatif, en incluant M. Allii-populina.

D'autre part, nous étions obligés jusqu'à présent de conserver nos souches de Melampsora par « repiquage " toutes les deux semaines sur plants ou feuilles en survie, au laboratoire. Cette méthode contraignante ne permettait guère une production importante d'inoculum ni la constitution d'une collection de souches destinées aux tests de sélection pour la résistance.

Nous avons donc tenté de mettre à profit les capacités de survie des urédospores pour en déduire une méthode de conservation de longue durée.

\section{I. - Matériel et méthodes}

\section{1. - Récolte des spores}

Les spores ont été récoltées par grattage des urédospores de feuilles infectées (en nature ou au laboratoire) de $P$. x euramericana (Dode) Guinier cv. Robusta ( $M$. 
Larici-populina) et cv. Dorskamp (M. Allii-populina). Des contrôles au microscope ont permis de vérifier l'absence de mélange d'espèces de Melampsora dans les lots recueillis.

\section{2. - Conservation des spores}

A l'extérieur, la conservation en nature a été étudiée par entreposage de feuilles prélevées sur des plants infectés puis disposées en cages sur le sol de la pépinière de Champenoux.

Au laboratoire, les sporées ont été réparties dans des tubes de verre, fermés par du papier d'aluminium et regroupés dans des verrines au réfrigérateur $\left(+4^{\circ} \mathrm{C}\right.$, 85 p. 100 environ d'humidité relative). Dans une variante l'humidité relative a été ajustée en coulant au fond des verrines un mélange de sable et de solutions salines saturées : chlorure de magnésium, bromure de sodium et chlorure de sodium qui assuraient respectivement des humidités relatives de 34,63 et 75 p. 100 . Le sable ne servait qu'à empêcher les tubes (ouverts) de se renverser.

\section{3. - Contrôle de la germination}

Les fubes ont été utilisés à espaces de temps réguliers pour contrôler la perte de pouvoir germinatif. Les spores étaient alors mises en suspension dans de l'eau faiblement gélosée $(1$ p. 1000$)$ el pulvérisées aseptiquement sur de la gélose à 2 p. 100 en boîtes de Pétri ou disposées en gouttes pendantes sur des lamelles couvrant des cellules de Van Tieghem. Boîtes de Pétri ef cellules étaient placées 48 h sous éclairage fluorescent à température du laboratoire. Pour chaque modalité cinq cents spores, au moins étaient observées et seules celles dont le tube germinatif était plus long que la spore étaient considérées comme germées.

\section{II. - Résultats}

\section{1. - Survie en pépinière}

Les feuilles infectées par M. Allii-populina placées fin octobre en pépinière, y ont été retirées le 25 mai 1977. Les spores résiduelles ont été récupérées par grattage sous loupe binoculaire et mises en germination. Après 72 h, 3 p. 100 de spores germaient encore. Les conclusions de Taris (1968) sont donc généralisables à M. Allii-populina.

\section{2. - Survie au laboratoire}

La figure 1 traduit l'évolution du pouvoir germinatif des deux espèces après conservation au réfrigérateur. Seuls sont indiqués les pourcentages observés sur gélose. Ceux relatifs aux cellules de Van Tieghem onf toujours été légèremenf inférieurs. Les deux espèces confirment leur similitude de comportement. Le pouvoir germinatif décroît progressivement pour s'annuler après 7 ou 8 mois. L'augmentation initiale des pourcentages est un phénomène fréquent dont nous pensons qu'il traduit une phase de maturation des spores : le grattage détacherait des spores immatures. 


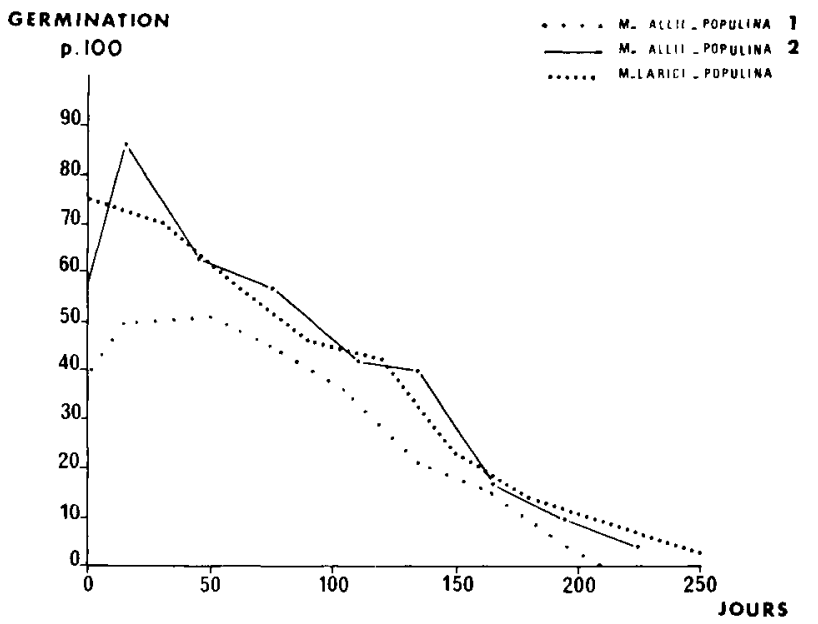

FIG. 1. Evolution du pouvoir germinatif des urédospores conservées $\grave{a}+4^{\circ} \mathrm{C}: \mathrm{M}$. Allii-populina $(1=$ spores collectées en pépinière, $2=$ spores produites au laboratoire) et $M$. Larici-populina (spores produites au laboratoire).

Evolution of germinability of uredospores stored at $+4^{\circ} \mathrm{C}: M$. Allii-populina $(1=$ spores collected in the nursery, 2 = spores produced in the laboratory) and $M$. Larici-populina (spores produced in the laboratory).

\section{GERMINATION}

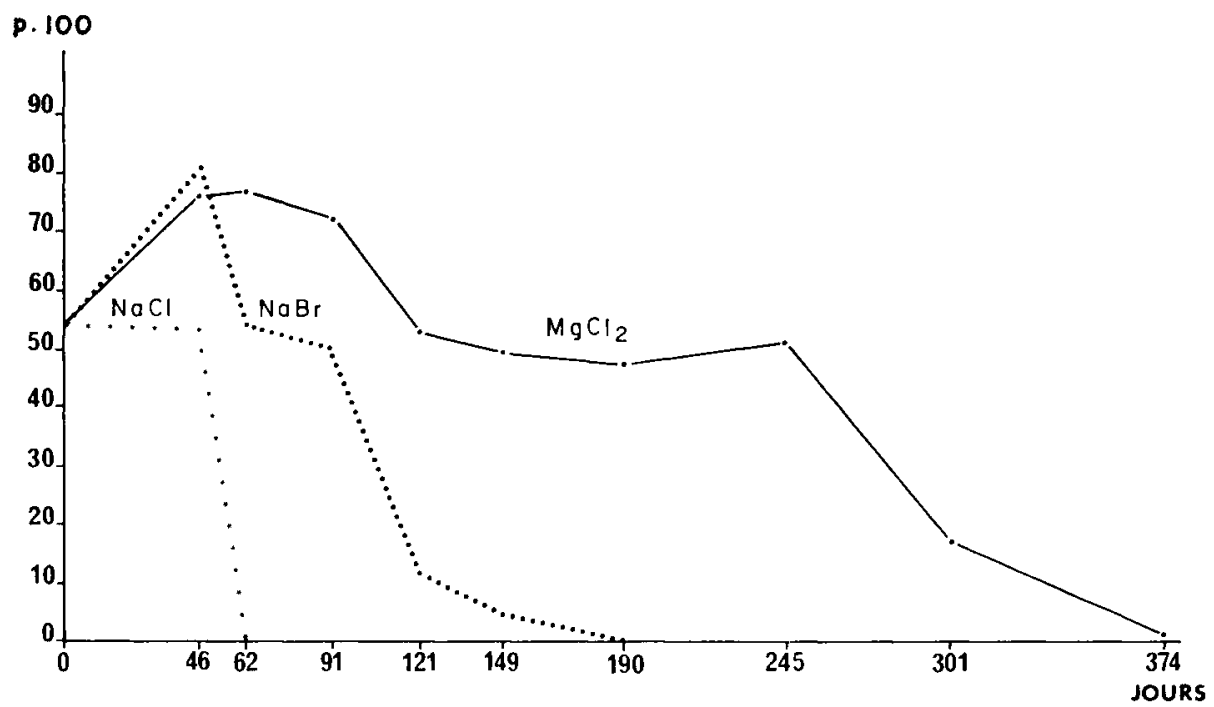

FIG. 2. - Conservation d'urédospores de M. Larici-populina collectées en pépinière et soumises d̀ diverses humidités relatives sous $+4^{\circ} \mathrm{C} . \mathrm{NaCl}=35$ p. $100, \mathrm{NaBr}=63$ p. $100, \mathrm{MgCl}_{2}=34$ p. 100.

Melampsora larici-populina uredospores conservation, when collected in the nursery and stored under various relative humidities of $+4^{\circ} \mathrm{C} . \mathrm{NaCl}=75$ p. $100, \mathrm{NaBr}=63$ p. $100, \mathrm{MgCl}_{2}=34$ p. 100 . 
Dans le cas de $M$. Allii-populina nous avons comparé deux lots d'origine différente. Les spores récoltées en pépinière présentent un plus faible pouvoir germinatif que celles obtenues au laboratoire après inoculation de feuilles en survie. Néanmoins la méthode de conservation à $+4^{\circ} \mathrm{C}$ conduit à une durée de survie des spores tout à fait comparable à celle déjà observée en pépinière.

La figure 2 récapitule l'ensemble des résultats relatifs au lot soumis à différentes humidités relatives. Dans le cas du bromure de sodium $(63 \mathrm{p} .100)$ et surtout du chlorure de sodium (75 p. 100) la survie est plus brève que celle notée à 85 p. 100 en l'absence de sels. Les différences modestes entre ces trois taux d'humidité ne peuvent expliquer ce phénomène que nous attribuons, à priori, à une toxicité de ces sels. A l'inverse, le chlorure de magnésium (34 p. 100) améliore nettement la survie. Après 374 jours quelques spores germent encore et durant les 8 premiers mois de conservation le taux de germination est demeuré supérieur à 50 p. 100.

\section{3. - Pouvoir pathogène}

Au terme de chacune des expériences précédentes nous avons pulvérisé des urédospores sur feuilles en survie. Il s'avère que même des lots de faible pouvoir germinatif résiduel in vitro (2-3 p. 100) étaient toujours pathogènes.

\section{Conclusion et discussion}

Les urédospores des deux espèces sont donc capables de survivre à l'hiver, de germer au printemps suivant, certes en modeste pourcentage, puis d'infecter les peupliers. Ceci confirme donc pour M. Allii-populina les conclusions de Taris relatives à M. Larici-populina. Ceci signifie que ces deux espèces de Melampsora peuvent très vraisemblablement se comporter en microcycliques et se multiplier végétativement au stade II.

D'un point de vue pratique nos souches ne devront plus subir qu'un repiquage annuel, comme en mycothèque classique. Nous pouvons ainsi envisager le clonage de souches possèdant des virulences données et donc fournir, pour les tests de sélection des spores de nature connue applicables, à des concentrations précises. Déjà en 1978, des urédospores produites en hiver au laboratoire ont été pulvérisées avec succès sur des lignes de plants contaminateurs lors d'un essai de sélection de peupliers résistants aux rouilles dans la pépinière du centre de Recherches forestières d'Orléans.

A l'avenir la méthode pourra être perfectionnée en estimant l'effet d'une gamme plus large d'humidités relatives et en nous adressant à des spores produites au laboratoire dont nous avons constaté un meilleur pouvoir germinatif.

Reçu pour publication en avril 1979. 


\section{Summary}

Longevity of uredospores of Melampsora Larici-populina Kleb and M. Allii-populina Kleb

Germinability of M. Larici-populina and M. Allii-populina decreases slowly during conservation at $+4^{\circ} \mathrm{C}$. As in the nursery, only a few spores still germ after 7 to 8 months. When temperature $\left(4^{\circ} \mathrm{C}\right)$ aswell as relative humidity (34 p. 100) are controlled, it becomes possible to keep spores alive for one year, with a high level of germination ( 50 p. 100) during 8 months.

\section{Références bibliographiques}

TARIS B., 1968. Contribution à l'étude des rouilles des Populus observées en France. Ann. Epiphyties, 19, 1-54. 